\title{
Multidrug resistant Pseudomonas aeruginosa in Estonian hospitals
}

\author{
Kaidi Telling ${ }^{1^{*}}$ (D, Mailis Laht ${ }^{2}$, Age Brauer ${ }^{3}$, Maido Remm³ ${ }^{3}$ Veljo Kisand ${ }^{2}$, Matti Maimets ${ }^{4}$, Tanel Tenson ${ }^{2}$ \\ and Irja Lutsar ${ }^{1}$
}

\begin{abstract}
Background: We aimed to identify the main spreading clones, describe the resistance mechanisms associated with carbapenem- and/or multidrug-resistant $P$. aeruginosa and characterize patients at risk of acquiring these strains in Estonian hospitals.

Methods: Ninety-two non-duplicated carbapenem- and/or multidrug-resistant $P$. aeruginosa strains were collected between 27th March 2012 and 30th April 2013. Clinical data of the patients was obtained retrospectively from the medical charts. Clonal relationships of the strains were determined by whole genome sequencing and analyzed by multi-locus sequence typing. The presence of resistance genes and beta-lactamases and their origin was determined. Combined-disk method and PCR was used to evaluate carbapenemase and metallo-beta-lactamase production.

Results: Forty-three strains were carbapenem-resistant, 11 were multidrug-resistant and 38 were both carbapenem- and multidrug-resistant. Most strains (54\%) were isolated from respiratory secretions and caused an infection (74\%).

Over half of the patients (57\%) were $\geq 65$ years old and $85 \%$ had $\geq 1$ co-morbidity; $96 \%$ had contacts with healthcare and/or had received antimicrobial treatment in the previous 90 days.

Clinically relevant beta-lactamases (OXA-101, OXA-2 and GES-5) were found in 12\% of strains, 27\% of which were located in plasmids. No Ambler class B beta-lactamases were detected. Aminoglycoside modifying enzymes were found in 15\% of the strains. OprD was defective in 13\% of the strains (all with CR phenotype); carbapenem resistance triggering mutations (F170 L, W277X, S403P) were present in 29\% of the strains. Ciprofloxacin resistance correlated well with mutations in topoisomerase genes gyrA (T83I, D87N) and parC (S87 L). Almost all strains (97\%) with these mutations showed ciprofloxacin-resistant phenotype.

Multi-locus sequence type analysis indicated high diversity at the strain level - 36 different sequence types being detected. Two sequence types (ST108 $(n=23)$ and ST260 $(n=18))$ predominated. Whereas ST108 was associated with localized spread in one hospital and mostly carbapenem-resistant phenotype, ST260 strains occurred in all hospitals, mostly with multi-resistant phenotype and carried different resistance genotype/machinery.
\end{abstract}

Conclusions: Diverse spread of local rather than international $P$. aeruginosa strains harboring multiple chromosomal mutations, but not plasmid-mediated Ambler class B beta-lactamases, were found in Estonian hospitals.

Trial registration: This trial was registered retrospectively in ClinicalTrials.gov (NCT03343119).

Keywords: Carbapenem resistance, Outbreak, WGS (whole-genome sequencing), Beta-lactamases

\footnotetext{
* Correspondence: kaidi.telling@ut.ee

${ }^{1}$ Department of Microbiology, Institute of Biomedicine and Translational

Medicine, University of Tartu, Ravila 19, 50411 Tartu, Estonia

Full list of author information is available at the end of the article
}

(c) The Author(s). 2018 Open Access This article is distributed under the terms of the Creative Commons Attribution 4.0 International License (http://creativecommons.org/licenses/by/4.0/), which permits unrestricted use, distribution, and reproduction in any medium, provided you give appropriate credit to the original author(s) and the source, provide a link to the Creative Commons license, and indicate if changes were made. The Creative Commons Public Domain Dedication waiver (http://creativecommons.org/publicdomain/zero/1.0/) applies to the data made available in this article, unless otherwise stated. 


\section{Background}

Pseudomonas aeruginosa (PA) is an opportunistic pathogen present in many ecological settings. It can survive in living (humans, animals, plants) and non-living (water, soil, artificial surfaces) sources [1], but is rarely found in the microbiota of healthy humans [2]. The colonization rate by PA significantly increases (reaching up to $80 \%$ ) in patients with chronic illnesses (e.g. cystic fibrosis, severe burns) or extensive exposure to healthcare facilities involving interruption of protective barriers [3-5].

PA is exceptionally flexible, using different regulatory and metabolic mechanisms to adapt to antibiotic pressure. It has an intrinsic resistance to wide range of antimicrobial agents, and a high capacity to attain resistance mutations and mobile genetic elements [6].

According to the European Centre for Disease Prevention and Control (ECDC) in 2016, resistance of PA in most European countries exceeded $10 \%$ of all antimicrobials investigated. Furthermore, the prevalence of MDR-PA is rising globally, a phenomenon mainly associated with the spread of high-risk clones (e.g. multi-locus sequence types ST235, ST111, ST175) associated with nosocomial outbreaks and transferable resistance mechanisms, especially horizontally-acquired beta-lactamases [7]. Despite the low antibiotic consumption comparable to other North European countries [8], the resistance rates of PA in Estonia, especially to carbapenems, are much higher than in other low-end usage countries, with the trends becoming alarming. In 2012, $12.5 \%$ of the strains reported to the ECDC were carbapenem-resistant (CR), but this had risen above $20 \%$ by $2016[9,10]$.

Although main trigger associated with $\mathrm{CR}$ in PA is production of plasmid-mediated beta-lactamases/carbapenemases, mutational resistance mechanisms in chromosomal genes - e.g. altered expression of outer membrane porins or efflux systems and increased chromosomal cephalosporinase (AmpC) activity, may all have affected the development of resistance [7].

Unfortunately data provided by the ECDC reflects just a fraction of the actual situation, as only invasive strains and the phenotypic resistance are reported. This leaves a gap in terms of non-invasive infections, and in genetic information of the spreading bacterial lineages and resistance machinery they carry. Previous studies conducted in Estonia are no exception; they have included only certain patient groups (intensive care units) [11] or blood-stream infections [12] without any information at the molecular level. Both knowledge of main risk groups and genetic data of strains is essential in understanding resistance transfer, and to take actions needed to stop it.

\section{Methods}

We aimed to characterize hospitalized patients carrying carbapenem- or/and multidrug- resistant $P$. aeruginosa
(CR/MDR-PA), identifying the main spreading clones and describing the most important resistance mechanisms, including the occurrence of clinically relevant beta-lactamases.

\section{Study design and settings}

The study was conducted in 5 major Estonian hospitals treating both pediatric and adult patients - 2 3rd level multidisciplinary referral hospitals (Tartu University Hospital and North Estonia Medical Centre), 2 central hospitals (West Tallinn Central Hospital and East-Tallinn Central Hospital) and 1 private hospital specializing in plastic and vascular surgery (The Hospital of Reconstructive Surgery).

During the study period all consecutive PA strains isolated from hospitalized patients taken by discretion of treating physicians on suspicion or confirmed infection and identified as CR and/or MDR by the microbiology laboratories of the hospitals were collected, with a target of 100 strains. Only one strain was included from each patient (invasive strain or firstly isolated strain). Strains from ambulatory patients and clinics were excluded. The collection lasted from 27th of March 2012 to 30th of April 2013.

\section{Clinical data collection}

Hospital records of patients with eligible PA strains were reviewed retrospectively to obtain demographic data (age, gender), the presence of major co-morbidities and predisposing clinical conditions, date and reason of admission, site of infection, in-hospital movement, presence of infection or colonization caused by CR/MDR-PA, and the outcome.

Specifically, we recorded the presence of invasive devices, surgery within the previous 30 days, hospitalization, or time spent in a long-term care facility, and antimicrobial therapy in the previous 90 days.

\section{Sampling and microbiological methods}

Standard clinical laboratory methods were used to isolate and identify PA from clinical specimens. Briefly, urine was plated on cysteine lactose electrolyte-deficient agar, respiratory samples on blood and chocolate agar, which were incubated at $37{ }^{\circ} \mathrm{C}$ for 24 to $48 \mathrm{~h}$. Tissue biopsies were homogenized and incubated in thioglycolate broth at $37{ }^{\circ} \mathrm{C}$ for 14 days. Blood, cerebrospinal, pleural and abdominal fluids were processed and monitored with a BACTEC 9240 blood culture system (Becton Dickinson, Sparks, MD, USA). One colony with Pseudomonas-like morphology was identified using classical biochemical tests (catalase and oxidase reactions) and VITEK2 Compact or API tests (bioMérieux, Marcy l'Etoile, France).

Finally, all PA isolates were confirmed by using matrix-assisted laser desorption ionization time-of-flight mass spectrometry (Bruker Daltonics, Bremen, Germany). 
Only one isolate per patient was included in the final analysis, giving preference to more invasive strains (blood or cerebrospinal fluid).

\section{Phenotypic susceptibility testing}

The MIC for 9 antipseudomonal antibiotics (ceftazidime, cefepime, meropenem, imipenem, piperacillin/tazobactam, amikacin, gentamicin, tobramycin and ciprofloxacin) for each strain was measured with an epsilometer (E-test, bioMérieux, Marcy l'Etoile, France), with the quality control strain ATCC ${ }^{\oplus} 27,853^{\mathrm{TM}}$ being routinely used. Antibiotic susceptibility was determined using EUCAST breakpoints and definitions [13, 14]. Strains non-susceptible to at least one tested carbapenem were designated CR, whereas those not susceptible to 3 or more antibiotic classes were defined as MDR [13].

Forty-seven CR strains were screened for Klebsiella pneumoniae carbapenemase (KPC) and metallo-beta-lactamase (MBL) production by combined-disk method using disks containing $10 \mu \mathrm{g}$ imipenem, $10 \mu \mathrm{g}$ imipenem with phenylboronic acid, $10 \mu \mathrm{g}$ imipenem with cloxacillin high, $10 \mu \mathrm{g}$ imipenem with dipicolinic acid and imipenem with EDTA (Rosco Diagnostica, Taastrup, Denmark). Quality control strains were $P$. aeruginosa CCUG59626, K. pneumoniae BAA1705 and E. coli ATCC $25,922^{\text {TM }}$.

PCR-based test for detection of genes encoding carbapenemases was used according to Poirel et al. [15] on the same 47 strains for double-control. Three different multiplex reaction mixtures were defined and evaluated for the detection of MBL-encoding genes ( $\boldsymbol{b l a}_{\mathrm{IMP}}$, $\boldsymbol{b l a}$ VIM, $\boldsymbol{b} \boldsymbol{l a}_{\mathrm{GIM}}$ and $\left.\boldsymbol{b} \boldsymbol{l} \boldsymbol{a}_{\mathrm{NDM}}\right)$, class A carbapenemase gene $\boldsymbol{b l a}_{\mathrm{KPC}}$ and class D carbapenemase gene $\boldsymbol{b l a}_{\mathrm{oxa}-48}$. The following control strains were used - Swedish Institute for Communicable Disease Control (Sweden) carbapenemases detection control set: OXA-48-positive K. pneumoniae Oxa241, KPC-positive K. pneumoniae K271, NDM-1-positive $K$. pneumoniae K275, IMP-positive $P$. aeruginosa CCUG59626, VIM-positive $K$. pneumoniae CCUG58547, GIM-positive P. aeruginosa; and KPC-positive K. pneumoniae BAA1705.

\section{DNA extraction}

A modified GuSCN-silica protocol was used for the DNA extraction from a single colony [16]. Briefly, cells were transferred into a solution containing $570 \mu \mathrm{l}$ TE (pH 7.6) buffer (TRIS-EDTA) and $30 \mu \mathrm{L} 10 \%$ SDS, with $\sim 0.5$ g zirconium beads ( $0.1 \mathrm{~mm}$ diameter), which was processed by $5 \mathrm{~min}$ on bead beater (Minibead beater, Bio-Spec Products, Bartlesville, USA), followed by centrifugation at $10000 \mathrm{rpm}$ for $1 \mathrm{~min}$. Cells were lysed by combining the supernatant with lysis buffer L6 (5.25 M GuSCN, 100 mM Tris - HCl, pH 6.4, 20 mM EDTA, $1.3 \%$ Triton $\mathrm{X}-100)$. Custom-prepared silica suspension $(40 \mu \mathrm{l})$ was added before incubation for $5 \mathrm{~min}$ at room temperature and centrifugation at $5000 \mathrm{rpm}$ for $10 \mathrm{~s}$. The supernatant was discarded and the pellet washed with buffer L2 (5 M GuSCN) and 50\% ethanol. The silica pellet was briefly dried and the DNA eluted in ultra-pure water (milli-Q). The extracted DNA was stored at $-20{ }^{\circ} \mathrm{C}$ until analyzed.

\section{Whole genome sequencing}

Total bacterial DNA was quantified using a Qubit ${ }^{\bullet} 2.0$ Fluorometer (Invitrogen, Grand Island, USA) and 2200 TapeStation (Agilent Technologies, Santa Clara, USA). Ten nanograms of sample DNA was processed using an Illumina Nextera XT sample preparation kit (Illumina, San Diego, USA). The resulting DNA libraries were validated by qPCR using a Kapa Library Quantification Kit (Kapa Biosystems, Woburn, USA) to optimize cluster generation.

Ninety-two ssDNA Nextera XT libraries originating from 92 different clones were pooled and sequenced in one rapid-output run of HiSeq2500 (Illumina, San Diego, USA), with paired-end, 150-bp reads. Demultiplexing was done with CASAVA 1.8.2. (Illumina, San Diego, USA), allowing one mismatch in the index reads.

\section{Draft assembly of whole genome sequences (WGS), multi-} locus sequence typing (MLST) and phylogeny analysis

All Illumina reads were assembled de novo using the SPAdes genome assembler (ver 3.5.0), together with the MismatchCorrector [17].

A BLAST-based tool from https://cge.cbs.dtu.dk/services/MLST/ was run to annotate the MLST fragments within the WGS data [18]. To identify the sequence types (ST), the batch profile query from the pubMLST website for PA (http://pubmlst.org/paeruginosa) together with their locus/sequence definitions was used.

Sequences were aligned using global genome alignment to determine the core genomes. Thereafter, recombinations in the core genomes were detected using BratNextGen software [19]. For phylogenetic analysis, recombination-free alignments were created by masking all significant recombinant segments as missing data in the input alignment. These alignments were used to reconstruct a maximum likelihood phylogenetic tree with RaxML, using the GTR-GAMMA model.

As core genome alignment and MLST analysis resulted in similar clustering, the data are presented according to STs of MLST.

\section{Identifying resistance genes}

Antibiotic resistance genes were identified using a homology search against the collection of antibiotic resistance protein sequences from The Comprehensive Antibiotic Resistance Database (CARD, http://arpcard.mcmaster.ca/, version 1.1.0) and beta-lactamases from http://www.lahey.org/ 
studies/. GyrA, ParC, OprD, Rmt and Arm sequences were retrieved from the NCBI protein database. Identity and coverage thresholds were set to $90 \%$.

Clinical relevance of found beta-lactamases was assessed as suggested by Potron et al. [20]

The chromosomal or plasmid origin of beta-lactamase genes was determined using a blastn search of corresponding contigs against the NCBI nt/nr database. Top matches were also examined manually to determine whether they were plasmid or chromosomal sequences. We could not decide whether the beta-lactamase gene was located in plasmid or chromosome for very short contigs and/or contigs with low coverage matches, or matches against both chromosomal and plasmid sequences. Contigs matching only against chromosomal genome sequences were not considered to be plasmid-related, whereas contigs matching with high coverage $(>95 \%)$ and identity (>98\%) to only plasmid sequences were considered as possibly originating from the plasmids.

\section{Definitions and statistical analysis}

For the analysis of demographic and clinical characteristics, patients were categorized as infected and colonized according to following criteria. Colonization was defined as absence of clinical signs of infection on day of isolation $\mathrm{PA}$ in the anatomical site were microorganism was detected. Infections were classified by their most probable origin of acquisition to community and hospital-acquired. ECDC definitions for hospital-acquired infections were used [21]. The Charlson weighted index of comorbidity was calculated using an updated (ICD-10 diagnosis-based) version [22].

Descriptive analysis used R 2.8.1 [23]. Chi-square or Fisher's exact tests were run where appropriate for categorical and the Kolmogorov-Smirnov test for continuous variables. Adjustment for multiple testing was made using the Bonferroni method.

\section{Results}

The local microbiology laboratories identified a total of 118 CR/MDR-PA strains, of which 26 were excluded from the final analysis ( 16 from ambulatory patients, 6 were duplicates from the same patient, and 4 were not PA according to MALDI-TOF). Of the 92 strains, 43 were CR, 11 were MDR, and 38 were both MDR and CR.

The most frequently represented sources were respiratory secretions $(n=50 ; 54 \%)$, followed by wound aspirates ( $n=22 ; 24 \%)$, urine $(n=12 ; 13 \%)$ and materials retrieved during intra-abdominal or vaginal procedures or surgeries $(n=7 ; 8 \%)$. One strain originated from the bloodstream.

The median time elapsed between hospital admission and CR/MDR-PA isolation was 13 days (IQR: 6-27 days).

\section{Study population and characteristics}

Sixty-eight (74\%) patients had infections, whereas 24 $(26 \%)$ were classified as asymptomatic carriers. The most common infections were pneumonia $(n=34 ; 50 \%)$, skin and soft tissue $(n=14 ; 21 \%)$, surgical site $(n=6 ; 9 \%)$, and intra-abdominal infections $(\mathrm{n}=5 ; 7 \%)$. About half the patients $(n=44 ; 47 \%)$ had hospital-acquired infections (Additional file 1: Table S1).

The demographic and clinical characteristics of patients that had CR/MDR-PA are presented in Table 1.

Over half of the patients were elderly ( $\geq 65$ years) with multiple co-morbidities (average $( \pm \mathrm{SD})$, Charlson comorbidity index $(2.6 \pm 2.1)$. About $85 \%$ patients had at least one co-morbidity. There were no patients $<18$ years old.

Congestive cardiac insufficiency was commonly present (32\%), followed by renal failure (23\%) and diabetes mellitus $(22 \%)$. Although 17 patients had chronic pulmonary disease, none had cystic fibrosis.

Most of the patients ( $n=88 ; 96 \%)$ had preceding contact with the healthcare and/or received antibacterial treatment within the previous 90 days; beta-lactams were by far the most commonly used antibiotics (Additional file 2: Table S2).

There were no statistically significant differences detected in risk factors of colonized and infected patients probably due to small sample size and great variety in population analyzed. It might be assumed that factors affecting exposure to PA and leading from colonization to infection are quite diverse and need further research in more precisely selected groups to draw adequate conclusions.

Eighteen patients (20\%) died during their hospital stay. The mortality rates in the 7 day, 30 day and 1 year after PA isolation were 2,14 and $44 \%$, respectively.

\section{Antibiotic susceptibility of PA}

The MIC values together with the interpretations are shown in Table 2. The highest resistance rates were observed to imipenem and the lowest to amikacin (59.8 and $7.6 \%$, respectively).

CR was detected in 81 (88\%) strains but no production of KPC or MBL was found by using combined-disk method and PCR in 47 randomly selected CR strains (Fig. 1).

\section{Genetic relationship and spread}

Ninety-two CR/MDR-PA strains were assigned to 36 different sequence types (Fig. 1), of which 9 strains were novel to the MLST database.

The most prevalent sequence types were ST108 $(n=23$; $25 \%)$ and ST260 ( $n=18 ; 20 \%)$. ST108 strains were found throughout the study, indicating an endemic spread in 6 different wards (mostly in 2 ICUs) of one hospital. Eleven patients carrying ST108 had nosocomial infection, mostly 
Table 1 The most important clinical characteristics of patients with CR/MDR-PA infection or colonization

\begin{tabular}{|c|c|c|c|c|}
\hline & All $(n=92)$ & Infection $(n=68)$ & Colonization $(n=24)$ & OR $(95 \% \mathrm{Cl})$ \\
\hline \multicolumn{5}{|l|}{ Demographic characteristics } \\
\hline Male sex (\%) & $56(61)$ & $44(65)$ & $12(52)$ & $1.8(0.7-4.7)$ \\
\hline Age: median; years (IQR) & $68(52-74)$ & $69(51-74)$ & $66(52-75)$ & \\
\hline Patients $\geq 65$ years (\%) & $52(57)$ & $37(54)$ & $15(63)$ & $0.7(0.3-1.9)$ \\
\hline \multicolumn{5}{|l|}{ Previous contact with health care system } \\
\hline Hospitalization within 90 days (\%) & $35(38)$ & $25(37)$ & $10(42)$ & $0.8(0.3-2.1)$ \\
\hline Long-term healthcare facilities stay within 90 days (\%) & $5(5)$ & $4(6)$ & $1(4)$ & $1.4(0.2-13.5)$ \\
\hline Antibiotic therapy in previous 90 days (\%) & $82(89)$ & $60(88)$ & $22(92)$ & $0.7(0.1-3.5)$ \\
\hline Surgery in previous 30 days (\%) & $53(58)$ & $38(56)$ & $15(63)$ & $0.8(0.3-2.0)$ \\
\hline Intensive care unit stay; duration in days: median (IQR) & $57(62) ; 15(8-24.5)$ & $40(59)$ & $17(71)$ & $0.6(0.2-1.6)$ \\
\hline \multicolumn{5}{|l|}{ Invasive procedures in previous 30 days } \\
\hline Bronchoscopy & $30(33)$ & $25(37)$ & $5(21)$ & $2.2(0.7-6.7)$ \\
\hline Hemodialysis & $17(19)$ & $13(19)$ & $4(17)$ & $1.2(0.3-4.1)$ \\
\hline Endoscopy & $17(18)$ & $14(21)$ & $3(13)$ & $1.8(0.5-7.0)$ \\
\hline Other & $16(17)$ & $10(15)$ & $6(25)$ & $0.5(0.2-1.6)$ \\
\hline \multicolumn{5}{|l|}{ Invasive device use on the microorganism isolation day } \\
\hline Mechanical ventilation of the lungs & $47(51)$ & $34(50)$ & $13(54)$ & $0.8(0.3-2.2)$ \\
\hline Central venous catheter & $48(52)$ & $33(49)$ & $15(63)$ & $0.6(0.2-1.5)$ \\
\hline Urinary catheter & $51(55)$ & $38(56)$ & $13(54)$ & $1.1(0.4-2.7)$ \\
\hline Epicystostomy & $9(10)$ & $7(10)$ & $2(8)$ & $1.3(0.2-6.5)$ \\
\hline \multicolumn{5}{|l|}{ Co-morbidities } \\
\hline Congestive cardiac insufficiency & $29(32)$ & $21(31)$ & $8(33)$ & $0.9(0.3-2.4)$ \\
\hline Chronic renal failure & $21(23)$ & $16(24)$ & $5(21)$ & $1.2(0.4-3.6)$ \\
\hline Diabetes mellitus & $20(22)$ & $15(22)$ & $5(21)$ & $1.1(0.3-3.4)$ \\
\hline Chronic pulmonary disease & $17(18)$ & $12(18)$ & $5(21)$ & $0.8(0.3-2.6)$ \\
\hline Malignant tumor & $16(16)$ & $9(13)$ & $7(25)$ & $0.4(0.1-1.1)$ \\
\hline Hemiplegia & $14(16)$ & $11(16)$ & $3(13)$ & $1.4(0.3-5.3)$ \\
\hline Myocardial infarct & $13(14)$ & $9(13)$ & $4(17)$ & $0.8(0.2-2.8)$ \\
\hline Cerebrovascular disease & $12(13)$ & $11(16)$ & $1(4)$ & $4.4(0.5-36.4)$ \\
\hline Other $^{a}$ & $34(37)$ & $27(40)$ & $7(29)$ & $1.6(0.6-4.4)$ \\
\hline
\end{tabular}

${ }^{\mathrm{a} H I V}$, AIDS, hematologic malignancy, dementia, connective tissue disease, liver disease, peripheral vascular disease, ulcer disease, neutropenia, trauma, burn

ventilator-associated pneumonia (10 cases). All ST108 strains were CR (22 resistant to imipenem and 18 to meropenem) and 9 strains were MDR.

ST260 was found in all participating hospitals, but almost half of the strains $(n=7 ; 39 \%)$ belonging to this ST were isolated from a single hospital that was linked to one ICU. In most of cases $(n=14 ; 78 \%)$ ST260 caused infection and was phenotypically tested as MDR.

Only one strain belonged to the so-called high-risk international clone (ST235).

\section{Resistance genes}

Beta-lactamases

Clinically relevant beta-lactamases (OXA-101, OXA-2 and GES-5) were found in $12 \%$ of the strains. Oxacillinases belonging to class $\mathrm{D}$ were found in 10 isolates, with OXA-101 ( $n=9 ; 10 \%)$ being the most frequent and isolated only in ST260. No class B beta-lactamases (IMP, VIM; SPM; GIM, NDM, FIM) were present. None of the strains produced carbapenemases.

Plasmid encoded $\beta$-lactamases were present in 3 (3\%) of the strains. Only one plasmid encoded class A beta-lactamase, GES-5; an isolate that belongs to the previously well-known high-risk, clone ST235.

\section{Aminoglycoside modifying enzymes (AME) and 165 rRNA methyltransferases}

Clinically relevant AME genes were found in 14 (15\%) strains, the most frequent being $\operatorname{ANT}\left(2^{\prime \prime}\right)$-Ia, present in 11 (12\%) strains, 9 of which belonged to ST260. 
Table 2 Minimal inhibitory concentrations and antibiotic susceptibility interpretations of PA $(n=92)$

\begin{tabular}{|c|c|c|c|c|c|c|}
\hline Antibiotic & MIC range (mg/L) & $\mathrm{MIC}_{50}(\mathrm{mg} / \mathrm{L})$ & $\mathrm{MIC}_{90}(\mathrm{mg} / \mathrm{L})$ & S (\%) & I (\%) & $\mathrm{R}(\%)$ \\
\hline \multicolumn{7}{|l|}{ Beta-lactams } \\
\hline Imipenem & $0.5-32$ & 24 & 32 & 19.6 & 20.7 & 59.8 \\
\hline Piperacillin/tazobactam & $0.5-256$ & 16 & 256 & 50.0 & 0 & 50.0 \\
\hline Meropenem & $0.125-32$ & 8 & 32 & 28.3 & 25.0 & 46.7 \\
\hline Cefepime & $0.5-256$ & 6 & 32 & 70.7 & 0 & 29.3 \\
\hline Ceftazidime & $0.75-256$ & 2 & 64 & 73.9 & 0 & 26.1 \\
\hline Ciprofloxacin & $0.064-32$ & 0.5 & 32 & 53.3 & 0 & 46.7 \\
\hline \multicolumn{7}{|l|}{ Aminoglycosides } \\
\hline Gentamicin & $0.125-256$ & 2 & 48 & 80.4 & 0 & 19.6 \\
\hline Amikacin & $1-256$ & 6 & 16 & 75.0 & 17.4 & 7.6 \\
\hline Tobramycin & $0.125-256$ & 1 & 4 & 90.2 & 0 & 9.8 \\
\hline
\end{tabular}

Abbreviations: $M I C_{50}$ minimal inhibitory concentration inhibiting $50 \%$ of isolates, $M I C_{90}$ minimal inhibitory concentration inhibiting $90 \%$ of isolates, $S$ susceptible, $I$ intermediately susceptible, $R$ resistant

The AAC(6')-Ib-cr cassette mediating resistance to both aminoglycosides and fluoroquinolones was found in one isolate belonging to ST235 phenotypically resistant to ciprofloxacin and amikacin, but sensitive to gentamicin and tobramycin.

No $16 \mathrm{~S}$ rRNA methyltransferase coding genes were found.

\section{Mutational resistance mechanisms}

The oprD gene was defective in 12 strains (13\%; 8 resistant to both imipenem and meropenem; 1 imipenem and 1 meropenem resistant, and 2 with carbapenem-susceptible) and mutated in 77 strains (84\%). We found 3 previously described mutations triggering $\mathrm{CR}-\mathrm{F} 170 \mathrm{~L}(n=18 ; 20 \%)$, S403P $(n=8$; 9\%) and W277X $(n=2 ; 2 \%)$. Statistical analysis showed an association between defective oprD and non-susceptibility to meropenem (9 meropenem resistant vs 3 meropenem sensitive strains; $p<0.05$ ).

Having researched fluoroquinolone resistance-associated mutations in topoisomerase genes gyrA (T83I, D87N) and parC (S87 L), we found T83I mutation was present in 27 (29\%) strains, of which 23 were ciprofloxacin resistant. D87N was found in one and parC S87 L substitution in 6 ciprofloxacin resistant strains. Both gyrA T83I and parC S87 L mutations correlated well with ciprofloxacin resistance ( 23 resistant vs 4 sensitive strains; $p<0.0001$, and 4 resistant vs 2 sensitive strains; $p<0.01$, respectively).

\section{Discussion}

This is the first study that addresses clinical risk factors alongside with the presence of resistance mechanisms in a mixed population of hospitalized patients infected or colonized with CR/MDR-PA and covers all major Estonian hospitals. The observations show that: (1) the vast majority of affected patients were elderly and had a history of previous contact with healthcare institutions and/or multiple co-morbidities confirming results of previous studies [24]; (2) the 2 predominant STs (ST108 and ST260) we described had good spreading potential and have rarely been recorded in previous studies, suggesting high ST variability within PA and minimal entry of internationally spreading strains into Estonian hospitals; (3) most of the CR strains lacked clinically relevant beta-lactamases including carbapenemases and metallo-beta-lactamases, suggesting that $C R$ is encoded by a selection of mutations in chromosomal genes; (4) correlation between gyrA T83I and parC S83 L mutations and quinolone resistance were in support of previous studies [25-27].

Considering the high rate of patients with advanced age, the high rate of contact with healthcare facilities, and association with multiple courses of prior antibiotic therapy, it is not unsurprising that they have been previously described as risk factors for PA infection [28]. However, none of these patients belonged to typical risk groups, such as burns, cystic fibrosis or febrile neutropenia, but more commonly had congestive cardiac insufficiency. Similarly, other studies have shown decreasing trends of PA in burn and oncologic patients in Europe and North-America [29]. The reason for these trends is not entirely clear, but the fact that most of the empirical treatment regimens include anti-pseudomonal antibiotics may be a contributing factor.

The high rate of patients with congestive heart disease is more difficult to explain. It may be a concomitant finding and not directly related to the colonization of CR/MDR-PA. This also highlights the fact that PA infection is not a disease strictly associated with people that have severe disturbances of immune or barrier systems, because it can also affect patients without obvious immune defects, such as the elderly. 


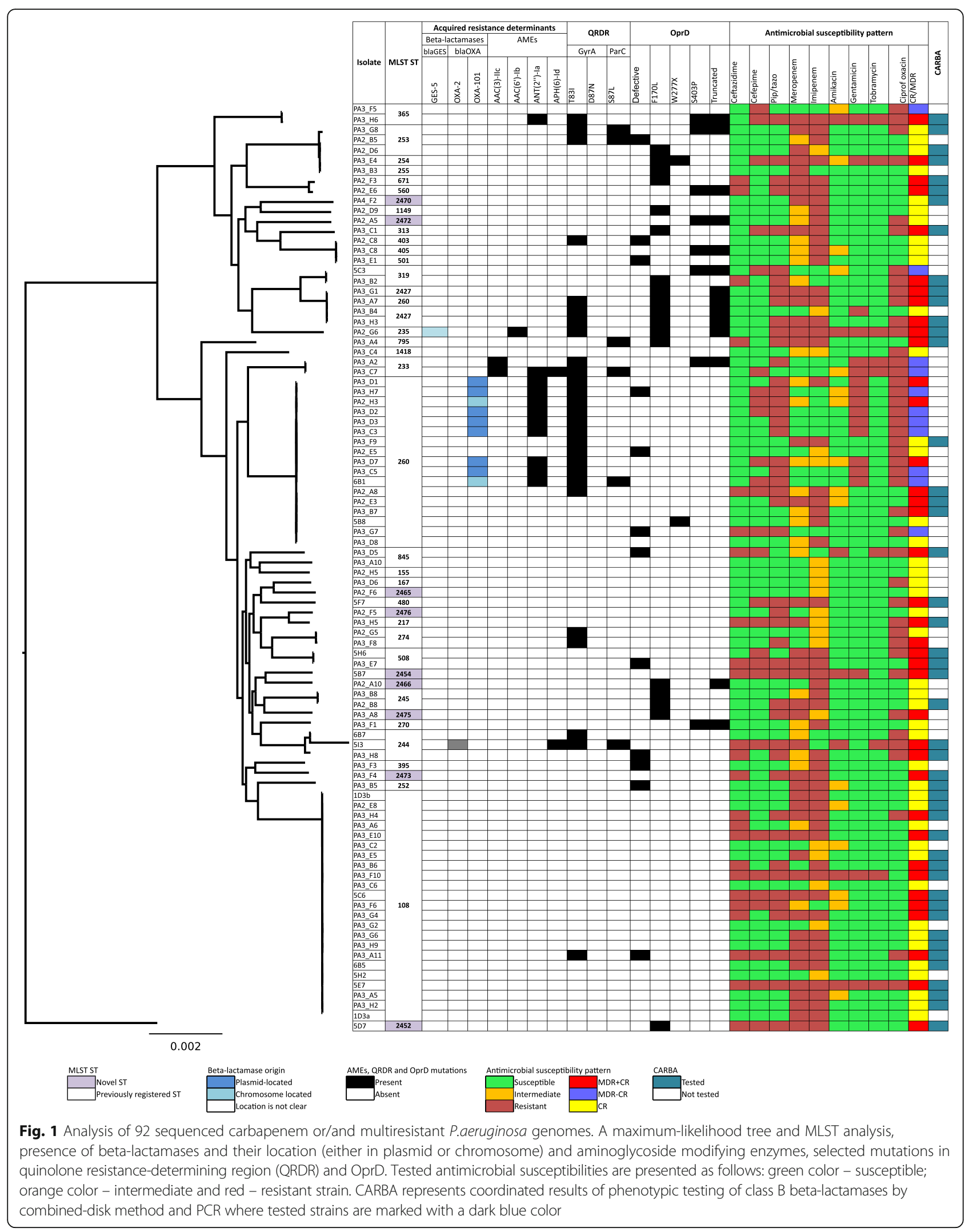


We found that the pattern of antibiotic resistance is largely driven but 2 STs (ST108 and ST265) causing hospital-acquired infections (mainly ventilator-associated pneumonia) and at least 2 outbreaks. Neither of these strains belonged to well-known internationally spreading clones [7]. ST108 was mostly associated with a singularly CR phenotype and spread only in one hospital. On the other hand, ST260 strains were much more diffused in hospitals. These strains usually had multiresistant phenotypes and carried some resistance machinery (ANT(2")-Ia, OXA-101 and GyrA T83I mutation). Hence ST may have the potential for becoming a new high-risk clone. ST260 has been previously described in different human settings and in different geographical regions; it is mostly associated with MDR or XDR phenotype, but never with outbreaks [30-33].

CR of PA, reaching up to $20.4 \%$ has been a problem within Estonian hospitals for years [34]; however, potential resistance mechanisms have remained unidentified [11], and unfortunately we do not have definite answers from this study. In contrast to other European studies in which the most important trigger of resistance is the production of horizontally-acquired beta-lactamases (mainly belonging to Ambler class B), we found none of these strains, only a few clinically relevant beta-lactamases, of which only one (GES-5) has been described as having carbapenemase activity [20]. We detected a correlation between defective $\operatorname{opr} D$ and meropenem-resistance in 10 strains and a single mutation previously associated with carbapenem resistance in 25 strains [27]. These mechanisms, however, did not explain resistant phenotype of the remaining 46 strains. Probably a cascade of mutations that were not addressed in this study, including structural modifications in AmpC, peptidoglycan recycling genes and mutations leading to efflux pump overexpression, are required to facilitate phenotypic resistance.

Our data indicates the importance of 2 locally spread resistant clones in spite of low antibiotic consumption; tackling the main spreading routes of these clones could significantly reduce the burden of CR/MDR-PA. Because both the spreading clones had been isolated from respiratory secretions and associated with mechanical ventilation or bronchoscopy, the procedures related to the maintenance of the airway should be the focal point in the prevention of colonization and infections with CR/MDR-PA. Updating both the knowledge and skills of basic hand hygiene and isolation methods, improving oral hygiene practices, and revising bronchoscope cleaning techniques, including disinfection processes, can be effective in aborting PA outbreaks [35]. Infection control measures could be significantly improved by implementing high resolution molecular identification techniques (egg. WGS, MLVA) into everyday practice for rapid detection of outbreaks and stopping the spread of multiresistant microorganism and thus should be encouraged [36].

Some limitations of our study should be noted. Because samples were identified by microbiology laboratories and taken on discretion of treating physicians, there is a possibility that some patients, especially asymptomatic carriers, were not sampled. This leaves a gap in our understanding of how MDR-PA circulates in Estonian hospitals. Secondly, E-test was used for susceptibility measurement instead of microdilution that is EUCAST suggested reference method. Still, E-test results have correlated well with MICs generated by the dilution methods [37] and thus we believe that our results are reliable. Despite these limitations we believe that our results allow us to draw adequate conclusions.

\section{Conclusions}

PA is a pathogen that affects not only immunocompromised, but also elderly multi-morbid patients. It is characterized by wide genetic diversity and spread via local rather than global clones in Estonian hospitals. The resistance machinery of PA is complex with only few certain correlations between genotypic and phenotypic resistance. Many different genetic changes may be required to develop the resistance pattern observed in phenotypic tests. High resolution genotyping methods are very valuable for tracking the spread of outbreaks, and therefore it is crucial to encourage the use of sequence-based methods in everyday practice.

\section{Additional files}

Additional file 1: Table S1. Characteristics of patients with hospitalacquired infections. (DOC $34 \mathrm{~kb}$ )

Additional file 2: Table S2. Antibiotic groups used in prior 90 days before resistant CR/MDR-PA isolation. (DOC $36 \mathrm{~kb}$ )

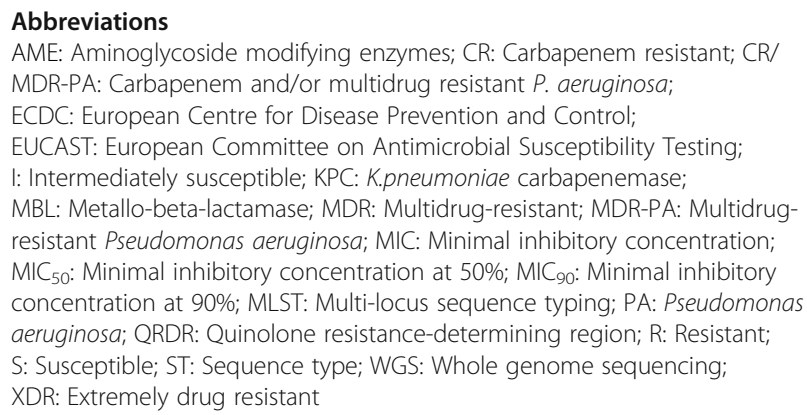

Acknowledgements

We are grateful to all infection control specialists and microbiologists involved in this project from the Tartu University Hospital, North Estonia Medical Centre, West Tallinn Central Hospital, and East-Tallinn Central Hospital. We are especially thankful to Anastasia Pavelkovich. Marina Ivanova, Paul Naaber and Tiiu Rööp for their help with quality control strains and methodology for carbapenemase testing. We thank BioMedES UK (www.biomedes.biz) for improvement of the final manuscript. 


\section{Funding}

This work was supported by Estonian Research Council (IUT2-22) and the European Union from the European Regional Development Fund through the Centre of Excellence in Molecular Cell Engineering and Estonian Program for Health Sciences (ABRESIST). Age Brauer and Maido Remm were funded by institutional grant IUT34-11 from the Estonian Ministry of Education and Research and the European Regional Development Fund through Centre of Excellence in Genomics and Translational Medicine (grant No. 20142020.4.01.15-0012). Veljo Kisand was funded by a personal research grant (PUT-134) from the Estonian Ministry of Education and Research. The funder had no role in the study design, data collection, analysis, decision to publish, or preparation of the manuscript.

\section{Availability of data and materials}

The datasets used and/or analyzed during the current study available from the corresponding author on reasonable request.

Novel sequences of the MLST alleles and new ST-s as combinations of previously reported allele sequences were reported to the pubMLST database (https://pubmlst.org/paeruginosa/, ID: BIGSdb_20170406141628_85178_50416). All WGS data have been deposited into the NCBI (PRJNA244279).

\section{Authors' contributions}

KT was a principal investigator and has contributed to all sections (implementation of study protocol, data collection, laboratory analyses, statistical analyses, first draft manuscript, final manuscript revision). ML participated in laboratory analyses and manuscript revision. AB did bioinformatical analysis, contributed to the results and discussion sections, as also to manuscript revision. MR gave bioinformatical advice and participated in manuscript revision. VK participated in laboratory analyses (WGS, MLST) and manuscript revision. MM contributed to developing the study protocol and revision of manuscript. $\Pi T$ and IL helped developing the study protocol, drafting first version of the manuscript, and revising all sections in the manuscript. All authors have read and approved the final manuscript

\section{Ethics approval and consent to participate}

The study protocol was approved with a waiver of informed consent by the Ethics Committee of the University of Tartu.

\section{Consent for publication}

Not applicable.

\section{Competing interests}

The authors declare that they have no competing interests.

\section{Publisher's Note}

Springer Nature remains neutral with regard to jurisdictional claims in published maps and institutional affiliations.

\section{Author details}

${ }^{1}$ Department of Microbiology, Institute of Biomedicine and Translational Medicine, University of Tartu, Ravila 19, 50411 Tartu, Estonia. ${ }^{2}$ Institute of Technology, University of Tartu, Tartu, Estonia. ${ }^{3}$ Institute of Molecular and Cell Biology, University of Tartu, Tartu, Estonia. ${ }^{4}$ Department of Infection Control, Tartu University Hospital, Tartu, Estonia.

\section{Received: 1 February 2018 Accepted: 27 September 2018}

\section{Published online: 11 October 2018}

\section{References}

1. Silby MW, Winstanley C, Godfrey SA, Levy SB, Jackson RW. Pseudomonas genomes: diverse and adaptable. FEMS Microbiol Rev. 2011;35(4):652-80.

2. Estepa V, Rojo-Bezares B, Torres C, Saenz Y. Faecal carriage of Pseudomonas aeruginosa in healthy humans: antimicrobial susceptibility and global genetic lineages. FEMS Microbiol Ecol. 2014;89(1):15-9.

3. Ciofu O, Tolker-Nielsen T, Jensen PO, Wang H, Hoiby N. Antimicrobial resistance, respiratory tract infections and role of biofilms in lung infections in cystic fibrosis patients. Adv Drug Deliv Rev. 2015;85:7-23.

4. Wolf SE, Phelan HA, Arnoldo BD. The year in burns 2013. Burns. 2014;40(8): $1421-32$.

5. Gomez-Zorrilla S, Camoez M, Tubau F, Periche E, Canizares R, Dominguez MA, Ariza J, Pena C. Antibiotic pressure is a major risk factor for rectal colonization by multidrug-resistant Pseudomonas aeruginosa in critically ill patients. Antimicrob Agents Chemother. 2014;58(10):5863-70.

6. Acharya M, Joshi PR, Thapa K, Aryal R, Kakshapati T, Sharma S. Detection of metallo-beta-lactamases-encoding genes among clinical isolates of Pseudomonas aeruginosa in a tertiary care hospital, Kathmandu, Nepal. BMC Res Notes. 2017;10(1):718.

7. Oliver A, Mulet X, Lopez-Causape C, Juan C. The increasing threat of Pseudomonas aeruginosa high-risk clones. Drug Resist Updat. 2015;21-22: 41-59.

8. Antimicrobial consumption database (ESAC-Net) [http://ecdc.europa.eu/en/ antimicrobial-consumption/surveillance-and-disease-data/database].

9. Antimicrobial resistance surveillance in Europe 2016 [http://ecdc.europa.eu/ en/publications-data/antimicrobial-resistance-surveillance-europe-2016].

10. Antimicrobial resistance surveillance in Europe 2012 [http://ecdc.europa.eu/ en/publications-data/antimicrobial-resistance-surveillance-europe-2012].

11. Loivukene K, Sepp E, Adamson V, Mitt P, Kallandi U, Otter K, Naaber P. Prevalence and antibiotic susceptibility of Acinetobacter baumannii, Pseudomonas aeruginosa and Klebsiella pneumoniae in Estonian intensive care units in comparison with European data. Scand J Infect Dis. 2006; 38(11-12):1001-8.

12. Mitt $\mathrm{P}$, Adamson V, Loivukene $\mathrm{K}$, Lang $\mathrm{K}$, Telling $\mathrm{K}$, Paro K, Room A, Naaber $P$, Maimets $M$. Epidemiology of nosocomial bloodstream infections in Estonia. J Hosp Infect. 2009:71(4):365-70.

13. Magiorakos AP, Srinivasan A, Carey RB, Carmeli Y, Falagas ME, Giske CG, Harbarth S, Hindler JF, Kahlmeter G, Olsson-Liljequist B, et al. Multidrugresistant, extensively drug-resistant and pandrug-resistant bacteria: an international expert proposal for interim standard definitions for acquired resistance. Clin Microbiol Infect. 2012;18(3):268-81.

14. EUCAST: Clinical breakpoints [http://www.eucast.org/clinical_breakpoints/]

15. Poirel L, Walsh TR, Cuvillier V, Nordmann P. Multiplex PCR for detection of acquired carbapenemase genes. Diagn Microbiol Infect Dis. 2011;70(1):119-23.

16. Boom R, Sol C, Wertheim-van Dillen P. Rapid purification of ribosomal RNAs from neutral agarose gels. Nucleic Acids Res. 1990;18(8):2195.

17. Bankevich A, Nurk S, Antipov D, Gurevich AA, Dvorkin M, Kulikov AS, Lesin VM, Nikolenko SI, Pham S, Prjibelski AD, et al. SPAdes: a new genome assembly algorithm and its applications to single-cell sequencing. J Comput Biol. 2012;19(5):455-77.

18. Larsen MV, Cosentino S, Rasmussen S, Friis C, Hasman H, Marvig RL, Jelsbak L, Sicheritz-Ponten T, Ussery DW, Aarestrup FM, et al. Multilocus sequence typing of total-genome-sequenced bacteria. J Clin Microbiol. 2012;50(4):1355-61.

19. Marttinen P, Hanage WP, Croucher NJ, Connor TR, Harris SR, Bentley SD, Corander J. Detection of recombination events in bacterial genomes from large population samples. Nucleic Acids Res. 2012;40(1):e6.

20. Potron A, Poirel L, Nordmann P. Emerging broad-spectrum resistance in Pseudomonas aeruginosa and Acinetobacter baumannii: mechanisms and epidemiology. Int J Antimicrob Agents. 2015;45(6):568-85.

21. Point prevalence survey of healthcare-associated infections and antimicrobial use in European acute care hospitals 2011-2012 [http://ecdc. europa.eu/en/publications/_layouts/forms/Publication_DispForm.aspx?List= 4f55ad51-4aed-4d32-b960-af70113dbb90\&ID=865].

22. Sundararajan V, Henderson T, Perry C, Muggivan A, Quan H, Ghali WA. New ICD-10 version of the Charlson comorbidity index predicted in-hospital mortality. J Clin Epidemiol. 2004:57(12):1288-94

23. R Core Team (2013). R: A language and environment for statistical computing. R Foundation for Statistical Computing, Vienna, Austria. URL http://www.R-project.org/. In.

24. Lin KY, Lauderdale TL, Wang JT, Chang SC. Carbapenem-resistant Pseudomonas aeruginosa in Taiwan: prevalence, risk factors, and impact on outcome of infections. J Microbiol Immunol Infect. 2016;49(1):52-9.

25. Kos VN, Deraspe M, McLaughlin RE, Whiteaker JD, Roy PH, Alm RA, Corbeil J, Gardner $\mathrm{H}$. The resistome of Pseudomonas aeruginosa in relationship to phenotypic susceptibility. Antimicrob Agents Chemother. 2015;59(1):427-36.

26. Cabot G, Ocampo-Sosa AA, Dominguez MA, Gago JF, Juan C, Tubau F, Rodriguez C, Moya B, Pena C, Martinez-Martinez L, et al. Genetic markers of widespread extensively drug-resistant Pseudomonas aeruginosa high-risk clones. Antimicrob Agents Chemother. 2012;56(12):6349-57.

27. Del Barrio-Tofino E, Lopez-Causape C, Cabot G, Rivera A, Benito N, Segura C, Montero MM, Sorli L, Tubau F, Gomez-Zorrilla S, et al. Genomics and Susceptibility Profiles of Extensively Drug-Resistant Pseudomonas aeruginosa Isolates from Spain. Antimicrob Agents Chemother. 2017:61(11): e01589-17. 
28. Cohen R, Babushkin F, Cohen S, Afraimov M, Shapiro M, Uda M, Khabra E, Adler A, Ben Ami R, Paikin S. A prospective survey of Pseudomonas aeruginosa colonization and infection in the intensive care unit. Antimicrob Resist Infect Control. 2017;6:7.

29. Mikulska M, Viscoli C, Orasch C, Livermore DM, Averbuch D, Cordonnier C, Akova M. Aetiology and resistance in bacteraemias among adult and paediatric haematology and cancer patients. J Inf Secur. 2014;68(4):321-31.

30. Chen SH, Chen RY, Xu XL, Chen HT. Multilocus sequencing typing of Pseudomonas aeruginosa isolates and analysis of potential pathogenicity of typical genotype strains from occupational oxyhelium saturation divers. Undersea Hyperb Med. 2014;41(2):135-41.

31. Mudau M, Jacobson R, Minenza N, Kuonza L, Morris V, Engelbrecht H, Nicol MP, Bamford C. Outbreak of multi-drug resistant Pseudomonas aeruginosa bloodstream infection in the haematology unit of a south African academic hospital. PLoS One. 2013;8(3):e55985.

32. Pobiega M, Maciag J, Chmielarczyk A, Romaniszyn D, Pomorska-Wesolowska M, Ziolkowski G, Heczko PB, Bulanda M, Wojkowska-Mach J. Molecular characterization of carbapenem-resistant Pseudomonas aeruginosa strains isolated from patients with urinary tract infections in southern Poland. Diagn Microbiol Infect Dis. 2015;83(3):295-7.

33. Samuelsen $\mathrm{O}$, Toleman MA, Sundsfjord A, Rydberg J, Leegaard TM, Walder M, Lia A, Ranheim TE, Rajendra Y, Hermansen NO, et al. Molecular epidemiology of metallo-beta-lactamase-producing Pseudomonas aeruginosa isolates from Norway and Sweden shows import of international clones and local clonal expansion. Antimicrob Agents Chemother. 2010;54(1):346-52.

34. European Centre for Disease Prevention and Control. Antimicrobial resistance surveillance in Europe 2016. Annual Report of the European Antimicrobial Resistance Surveillance Network (EARS-Net). Stockholm: ECDC; 2017.URL: http://ecdc.europa.eu/en/publications-data/antimicrobialresistance-surveillance-europe-2016

35. Sood G, Perl TM. Outbreaks in health care settings. Infect Dis Clin N Am. 2016;30(3):661-87.

36. Popovich KJ, Snitkin ES. Whole genome sequencing-implications for infection prevention and outbreak investigations. Curr Infect Dis Rep. 2017;19(4):15.

37. Reller LB, Weinstein $M$, Jorgensen JH, Ferraro MJ. Antimicrobial susceptibility testing: a review of general principles and contemporary practices. Clin Infect Dis. 2009:49(11):1749-55.

Ready to submit your research? Choose BMC and benefit from:

- fast, convenient online submission

- thorough peer review by experienced researchers in your field

- rapid publication on acceptance

- support for research data, including large and complex data types

- gold Open Access which fosters wider collaboration and increased citations

- maximum visibility for your research: over $100 \mathrm{M}$ website views per year

At BMC, research is always in progress.

Learn more biomedcentral.com/submissions 